\title{
Spezielle Aspekte der Ernährung bei Patienten mit abdomineller Sepsis
}

\author{
David Kuppinger Wolfgang $\mathrm{H}$. Hartl \\ Chirurgische Klinik und Poliklinik, Campus Großhadern, Ludwig-Maximilians-Universität München, Deutschland
}

Schlüsselwörter

Peritonitis · Schwere Sepsis · Ernährung · Leitlinie

\section{Zusammenfassung}

Hintergrund: Patienten mit schwerer abdomineller Sepsis stellen eine besondere ernährungsmedizinische Herausforderung dar. Methode: MEDLINE-, EMBASE- und Cochrane-Datenbanken wurden anhand der Stichwörter "peritonitis", "severe sepsis», "nutrition», "practice parameter» und "guideline» nichtsystematisch nach Publikationen durchsucht, die zwischen 2002 und 2012 erschienen waren. Wir berücksichtigten auch unser persönliches Literaturarchiv. Ergebnisse: Patienten mit abdomineller Sepsis besitzen ein hohes Risiko hinsichtlich einer schweren Hyperglykämie und Insulinresistenz. Aufgrund der gleichzeitig ausgeprägten und therapeutisch nur schwer zu beeinflussenden Eiweißkatabolie gelten diese Patienten als mangelernährt und benötigen eine besonders intensive Ernährungstherapie. Letztere orientiert sich nicht am tatsächlichen Energieumsatz (der vor allem in der Akutphase stark erhöht ist), sondern am Ausmaß der zu erwartenden Substratverwertungsstörungen. Ein zentraler Punkt ist dabei die Zufuhr von ausreichend Eiweiß oder Aminosäuren. Idealerweise sollte die Ernährung enteral erfolgen, wobei dies durch moderne Sonden- und Kathetertechniken bzw. operative Strategien auch bei schweren abdominellen Komplikationen (Anastomoseninsuffizienz) möglich ist. Allerdings zeigen Patienten mit abdomineller Sepsis oft eine verzögerte gastrointestinale Passage (Magen-/OberbauchAtonie, paralytischer Dünndarmileus, Pseudoobstruktion des Kolons), die durch engmaschige Überwachungsmaßnahmen erkannt werden muss und die die enterale Ernährung limitiert. Bei gastraler Ernährung stellt - neben dem klinischen Befund am Abdomen - die Messung des gastralen Residualvolumens den besten Kontrollparameter dar. Paralytische Passagestörungen sollten so aggressiv wie möglich therapiert werden. Im Vordergrund steht dabei die Sanierung des abdominellen Fokus, gefolgt von der Vermeidung antiperistaltisch wirksamer Medikamente und der Applikation properistaltisch wirksamer Substanzen bzw. physikalischer Maßnahmen. Schlussfolgerung: Die effiziente Ernährung von Patienten mit abdomineller Sepsis erfordert einen individuellen, patientenbezogenen Ansatz, der neben ernährungsmedizinischen auch besondere gastroenterologische Kenntnisse voraussetzt.
Keywords

Peritonitis · Severe sepsis · Nutrition · Guideline

\section{Summary}

Feeding Patients with Severe Abdominal Infections: Special Aspects

Background: Feeding patients with severe abdominal infections is particularly demanding. Method: The authors electronically searched the MEDLINE, EMBASE and Cochrane databases (using the keywords 'peritonitis', 'severe sepsis', 'nutrition', 'practice parameter', and 'guideline') and reviewed their personal databases for articles relevant to the issue which have been published between 2002 and 2012. Results: Patients suffering from abdominal sepsis are at a high risk for severe hyperglycemia and insulin resistance. Due to an excessive protein catabolism which cannot be overcome by standard nutritional therapy, these patients are malnourished and require a particularly careful nutritional support. The latter is not guided by the actual energy expenditure (which markedly increases during the acute phase) but by the capacity of the organism to utilize exogenous substrates (this capacity usually decreases during the acute phase). It is of outmost importance to supply sufficient amounts of protein or amino acids. Ideally, the patients should be fed enterally. Even in patients with severe abdominal complications (anastomotic leakage), modern tube and jejunostomy techniques as well as surgical strategies allow an adequate enteral supply of calories. However, patients suffering from a severe abdominal sepsis often exhibit a delayed gastrointestinal passage (delayed gastric emptying, small bowel paralysis, colonic pseudo-obstruction). These pathologies restrict enteral nutrition and should be recognized as early as possible by appropriate clinical surveillance. Besides a clinical examination of the abdomen, measurement of gastric residual volume represents the best control parameter when providing food into the stomach. Delayed gastrointestinal passage should be treated as aggressively as possible. Primary objective is the cure of the abdominal focus. In addition, use of drugs with an antiperistaltic action should be restricted as much as possible. Severe cases may profit from medications with a properistaltic action combined with specific physical measures. Conclusion: Feeding patients suffering from an abdominal infection requires an individualized, patient-centered approach which requires a profound nutritional and special gastroenterological knowledge.

\section{KARGER \\ Fax +497614520714 \\ Information@Karger.com}

www.karger.com (c) 2013 S. Karger GmbH, Freiburg

1662-6664/13/0291-0041\$38.00/0

Accessible online at:

www.karger.com/vim
Prof. Dr. Wolfgang H. Hart

Chirurgische Klinik und Poliklinik

Campus Großhadern, Ludwig-Maximilians-Universität München

Marchioninistraße 15, 81377 München, Deutschland

Wolfgang.Hart1@med.uni-muenchen.de 
minellen Sepsis (mit begleitenden Mehrfachorganversagen) kommt es dann zu einem Abfall des Energieumsatzes um 20-

Bei persistierend schwerer abdomineller Sepsis kommt es regelhaft zu einer gravierenden Störung des Kohlenhydratstoffwechsels mit Hyperglykämie und Insulinresistenz. Zum Teil sind unter solchen Umständen sehr hohe Kohlenhydratkonzentrationen zu beobachten, die, falls unbehandelt, über entsprechende immunsuppressive Effekte die Prognose des Patienten negativ beeinflussen können. Eine Progression der Insulinresistenz muss als Zeichen einer Progression des septischen Fokus angesehen werden [1,2].

Des Weiteren imponiert eine markante Eiweißkatabolie. Im Wesentlichen sind vier verschiedene Mechanismen für diesen protrahierten massiven Eiweißverlust verantwortlich, nämlich die Immobilisierung des Patienten, die anhaltende Ausschüttung von katabolen Hormonen und schließlich die infektgetriggerte Aktivierung bestimmter kataboler Zytokine. Letzterer Mechanismus führt zusätzlich zu einer peripheren Polyneuropathie und zu den Zeichen einer axonalen Degeneration (Critical-Illness-Polyneuropathie). In der Folge kommt es so zu einer funktionellen Denervierung und damit zu einer drastischen Erhöhung der Proteinabbaurate. Somit muss ein Patient mit schwerer abdomineller Sepsis (d.h. mit sekundärem Organversagen) immer als mangelernährt gelten. Schwer mangelernährte Patienten sind von einer erhöhten Morbidität betroffen und profitieren somit am meisten von einer adäquaten Ernährungstherapie [3-5].

Ein weiterer zentraler Befund bei fast allen Patienten mit schwerer abdomineller Sepsis ist die zunehmende Leberverfettung, die aus einer Imbalanz zwischen Fettsäureaufnahme, Fettsäureoxidation und Fettsäuregabe über die VLDL(very low density lipoprotein)-Triglyzeride resultiert. Aufgrund einer Repriorisierung im hepatischen Eiweißhaushalt beobachtet man eine gestörte hepatische VLDL-Triglyzeridsynthese bzw. -sekretion. Dieses Versagen der hepatischen Lipoproteinsynthese wird durch das translokationsinduzierte Bombardement der Leber mit intestinalen Toxinen verursacht und führt in Verbindung mit den vermehrt anflutenden freien Fettsäuren zur progredienten Einlagerung von Fett in das Lebergewebe sowie zu den unspezifischen Zeichen einer Cholangitis (Erhöhung von Gamma-GT- und alkalischen Phosphatase-Konzentrationen) [5, 6].

\section{Grundlagen der Ernährungstherapie bei abdomineller Sepsis}

\section{Abschätzung des Kalorienumsatzes}

Für den klinischen Alltag lässt sich der basale Umsatz einfach nach der Faustregel von Stein und Levine (basaler Energieumsatz (kcal)/pro Tag $=24 \times$ kg Körpergewicht) berechnen Dabei ist jedoch eine zeitliche Dynamik zu beobachten. In der Frühphase (nach akuter Fokus-Exazerbation) ist der Energieumsatz um $20-40 \%$ gesteigert. Im Vollbild der abdo-

$30 \%$. Nach erfolgreicher Fokussanierung bzw. im Stadium der Rehabilitation erhöht sich der basale Energieumsatz wieder um etwa $10 \%[5,7,8]$.

Die zeitliche Dynamik betrifft jedoch nicht nur den Energieumsatz, sondern auch die Fähigkeit des Organismus, zugeführte Substrate (speziell Kohlenhydrate und Fette) effektiv verwerten zu können. Das Ausmaß der Verwertungsstörung ist dabei indirekt proportional zur Aktivität des inflammatorischen Syndroms. Letzteres wird auch durch die bei abdomineller Sepsis oft nötigen Revisionseingriffe in den ersten postoperativen Tagen deutlich verstärkt.

\section{Substrate für die Ernährung}

Patienten mit abdomineller Sepsis werden entweder enteral oder parenteral ernährt. Dementsprechend existieren für diese Modalitäten kommerziell verfügbare Produkte. Die Produkte, die für Patienten mit abdomineller Sepsis zur Verfügung stehen und verwendet werden sollten, unterscheiden sich nicht von den Produkten für allgemeine Intensivpatienten. Der Einsatz dieser Produkte sollte entsprechend den Leitlinien [9-12] erfolgen.

\section{Praxis der Ernährungstherapie bei Patienten mit abdomineller Sepsis}

\section{Indikation und Modalität}

Bei Patienten mit abdomineller Sepsis sollte eine enterale Nahrungszufuhr so zügig wie möglich begonnen und entsprechend der gastrointestinalen Motilität sowie der Aktivität des inflammatorischen Syndroms sukzessive auf das ideale Kalorienniveau gesteigert werden [13, 14].

Eine parenterale Ernährung ist nur bei absoluten Kontraindikationen (Darmobstruktion mit relevanter Passagestörung, persistierendes intestinales Leck im mittleren Dünndarmdrittel, paralytischer Ileus, schwerer Schockzustand mit Kreislaufinstabilität) indiziert. In allen anderen Fällen wird zumindest der Versuch einer enteralen Ernährung empfohlen. Bei verlangsamter intestinaler Passage sollte zur Deckung des Kalorienbedarfs die künstliche Ernährung kombiniert enteral und parenteral erfolgen [15].

Auch bei Patienten, bei denen eine Anastomoseninsuffizienz am Gastrointestinaltrakt besteht, ist meistens eine enterale Ernährung möglich. Bei Anastomoseninsuffizienzen am Kolon und Rektum sollte in jedem Fall versucht werden, ein Stoma vorzuschalten, über das die verdaute Nahrung an der Insuffizienz vorbeigeleitet wird. Bei Anastomoseninsuffizienzen am oberen Gastrointestinaltrakt (Ösophagus/Magen/ Duodenum) ist die enterale Nahrungszufuhr über eine distal der Insuffizienz liegende transnasale Sonde bzw. Feinnadelkatheterjejunostomie zu empfehlen. Problematisch sind nur Patienten mit Leckagen im mittleren Dünndarmdrittel, das 
endoskopisch nicht zu erreichen ist. Bei diesen Patienten würde ein vorgeschaltetes Stoma bzw. eine distale Katheterjejunostomie $\mathrm{zu}$ einem Kurzdarmsyndrom führen. Somit ist unter solchen Bedingungen die parenterale Ernährung erforderlich [16].

Patienten mit insuffizienten Pankreas- oder Gallengangsanastomosen können problemlos enteral ernährt werden, da die Insuffizienz von der Nahrung nicht passiert wird. In solchen Situationen ist jedoch darauf zu achten, dass entweder Pankreasenzyme substituiert werden oder dass bei quantitativ hohem Galleverlust eine Rückführung der Galle aus der Insuffizienz über die Ernährungssonde in den Intestinaltrakt erfolgt.

\section{Höhe der Substratzufuhr}

Bei der Ernährungstherapie des Patienten mit abdomineller Sepsis ist - unabhängig von der Modalität der Zufuhr und vom Ausmaß des inflammatorischen Syndroms - der Schwerpunkt auf eine ausreichende Zufuhr von Eiweiß zu legen. Bei normalgewichtigen Patienten geht man von einem Bedarf von $1,5 \mathrm{~g} / \mathrm{kg}$ Körpergewicht und Tag aus. Die zusätzlich zu applizierenden Kohlenhydrat- bzw. Kohlenhydrat- und Fettmengen (dann idealerweise im Verhältnis 1:2) richten sich dann nach dem zuvor berechneten Energiebedarf bzw. den zu erwartenden Substratverwertungsstörungen [5, 11].

Gerade bei Patienten mit abdomineller Sepsis ist eine Steuerung der Kalorienzufuhr nach dem tatsächlichen Energieumsatz nicht angezeigt. Die Begründung ist darin zu suchen, dass auch eine sehr hohe Kalorienzufuhr die Katabolie der endogenen Substratspeicher nicht aufhalten kann und sich somit nicht metabolisch günstig auswirkt. Es können sogar eindeutig schädliche Nebeneffekte beobachtet werden. Bereits eine Erhöhung der Kalorienzufuhr um 20\% über den Ruheenergieumsatz hinaus führt bei Intensivpatienten so nur zu einer Zunahme der Fettmasse. Daher gilt für die überwiegende Mehrheit der septischen Patienten, dass eine konservative Kalorienzufuhr zwischen 21 und 25 kcal/kg Körpergewicht und Tag ausreicht (je nach Ausmaß der körperlichen Aktivität und Analgosedierung). Eine Kalorienzufuhr in derartiger Höhe stellt einen Kompromiss dar zwischen dem Versuch, körpereigene Verluste so weit wie möglich zu kompensieren, und dem Ziel, toxische Nebeneffekte zu vermeiden. Speziell bei akuter Exazerbation eines abdominellen inflammatorischen Syndroms sollte eine Reduktion der Kalorienzufuhr um 20-40\% (bezogen auf die unter diesen Umständen zu beobachtende Steigerung des Energieumsatzes) erfolgen. Die Reduktion bezieht sich dabei jedoch ausschließlich auf die zu applizierenden Fett- und Kohlenhydratmengen. Dabei sollte auch beachtet werden, dass eine Blutzuckerkonzentration von 180-200 mg/dl nicht überschritten wird [7, 17].

\section{Besonderheiten bei enteraler Kalorienzufuhr}

Grundvoraussetzung für jegliche Form der enteralen Therapie ist natürlich die suffiziente Passage und Resorption der zugeführten Substrate. Die tägliche Registrierung der tatsächlich zugeführten Substrat- und Flüssigkeitsmenge muss unter enteraler (speziell gastraler) Ernährung Bestandteil der Therapie sein. Insbesondere bei gastraler Ernährung über eine Sonde muss auf Passagestörungen geachtet werden. Gerade Patienten mit abdomineller Sepsis weisen ein hohes Risiko für eine eingeschränkte gastrointestinale Motilität auf. Diesbezüglich sind drei verschiedene Entitäten voneinander zu unterscheiden: i) die isolierte Oberbauchatonie (speziell anzutreffen bei entzündlichen Prozessen im Oberbauch), die die Magenentleerungs- und Motilitätsstörung im Duodenum und oberen Jejunum umfasst, ii) die Pseudoobstruktion des Kolons (Ogilvie-Syndrom) und iii) die globale intestinale Paralyse. Somit muss in der Anfangsphase der enteralen Ernährung und bei Verschlechterung des Allgemeinzustands eine regelmäßige klinische Evaluation des Abdomens und der Darmfunktion (Reflux/Obstipation/Durchfälle) erfolgen [17-20].

Zur enteralen Ernährung wird ein Zugang zum Gastrointestinaltrakt benötigt; dabei sind transnasale Sonden am einfachsten zu platzieren. Ist über einen absehbaren Zeitraum der obere Gastrointestinaltrakt nicht benutzbar, so sollte bereits intraoperativ eine Feinnadelkatheterjejunostomie angelegt werden. Zur nasogastralen Ernährung können großlumige Sonden (12-14 Charriere) verwendet werden, die gleichzeitig eine Dekompression des Magens erlauben.

Zwei verschiedene gastrale Applikationsmethoden - kontinuierliche Zufuhr oder Bolusgabe mit Portionen von 50-300 $\mathrm{ml}$ (mit oder ohne Gabe von Prokinetika) - sind grundsätzlich möglich. Allgemein zeigt die kontinuierliche Applikation eine bessere Toleranz mit höherer Energie- und Substratzufuhr, da hierunter die Raten an therapielimitierender Diarrhö und Aspirationsereignissen geringer sind. Andererseits können plötzlich auftretende Passagestörungen leicht übersehen werden, woraus sich in der Folge lebensbedrohliche Aspirationsereignisse entwickeln können. Bei unklarer Funktion (speziell bei Exazerbation eines abdominellen entzündlichen Fokus) sollte somit die manuelle Bolusapplikation zur Anwendung kommen, da das Pflegepersonal vor jeder erneuten Instillation das im Magen vorhandene Residualvolumen überprüfen und gegebenenfalls die zu applizierende Nahrungsmenge daraufhin reduzieren kann [17].

Als Surrogatvariable für gastrointestinale Motilitätsstörungen wird das gastrale Residualvolumen (GRV) verwendet. Zwei unterschiedliche Verfahren stehen zur Bestimmung des GRV zur Verfügung: Entweder wird die Magensonde mit einem Drainagebeutel konnektiert, geöffnet und der Drainagebeutel für 10 min unterhalb des Thoraxniveaus gelagert oder der residuale Mageninhalt wird mit einer 50-ml-Spritze über die Magensonde weitestmöglich aspiriert. Bei Patienten mit abdomineller Sepsis kommt hinsichtlich des GRV ein Schwellenwert von $200 \mathrm{ml}$ zur Anwendung. Übersteigt das GRV diesen Wert, so ist die gastrale Ernährung vorübergehend zu pausieren. Eine jejunale Ernährung muss allerdings 
nur dann pausiert werden, wenn gleichzeitig die klinischen bzw. radiologischen Zeichen einer Paralyse distaler Darmabschnitte bestehen [17, 20].

Die Überwachung der gastrointestinalen Motilität dient in der Summe drei Zwecken: Vorbeugung von pulmonalen oder intestinalen Komplikationen, Initiierung spezifischer therapeutischer Maßnahmen und Registrierung der tatsächlich zugeführten/mutmaßlich resorbierten Kalorien. Letzterer Punkt ist nicht zu vernachlässigen, da davon auszugehen ist, dass bei Patienten mit abdomineller Sepsis aufgrund gastrointestinaler Komplikationen oder Unterbrechungen der enteralen Ernährung aus anderer Ursache im Mittel nur etwa 50\% der verordneten Kalorien effizient enteral zuzuführen sind. Dies bedeutet für den Einzelfall, dass entsprechende Defizite genau gemessen und parenteral ausgeglichen werden müssen [17, 21].

Die Messung des GRV, der tatsächlich erfolgten Nahrungszufuhr und der Stuhlfrequenz/-art liegt üblicherweise in der Hand des Pflegepersonals, das diesbezüglich entsprechend ausgebildet sein sollte. Die klinische Überwachung des abdominellen Befundes ist in der Regel eine ärztliche Tätigkeit und hängt hinsichtlich ihrer Häufigkeit und Intensität vom Schweregrad der Grunderkrankung und von der Stabilität der gastrointestinalen Funktionen ab. Im Vordergrund steht dabei die körperliche Untersuchung, die bei pathologischen Befunden gegebenenfalls durch radiologische Maßnahmen ergänzt werden kann. Die Effizienz der enteralen Ernährung kann durch klar definierte therapeutische Algorithmen deutlich gesteigert werden $[18,21]$.

\section{Therapie von funktionellen Passagestörungen}

Die Therapie derartiger Störungen hat sich an den Ursachen $\mathrm{zu}$ orientieren. Da systemische inflammatorische Reaktionen die wichtigste Ursache darstellen, steht deren aggressive Therapie durch chirurgische und antimikrobielle Maßnahmen an erster Stelle. Die zweitwichtigste Ursache besteht in der Anwendung spezieller, paralytisch wirksamer intensivmedizinischer Therapien (Katecholamintherapie, Analgosedierung, $\beta_{2}$-Mimetika). Somit ist im Einzelfall zu überprüfen, welche dieser Therapien bei Präferenz eines funktionstüchtigen Intestinaltrakts eingeschränkt werden kann. Erst an dritter Stelle steht die symptomatische Therapie. Isolierte Oberbauchatonien können durch endoskopisches Einbringen einer nasojejunalen Sonde überbrückt werden. Zusätzlich können motilitätssteigernde Pharmaka (Metoclopramid oder Erythromyzin) zum Einsatz kommen. Die Paralyse distaler Darmabschnitte kann medikamentös auf zwei Arten beeinflusst werden. Erstens kann dies durch die Gabe von osmotisch wirksamen Substanzen, wie z.B. Meglumin-Amidotrizoat
(Gastrografin $^{\circledR}$; Bayer Vital GmbH, Leverkusen, Deutschland) oder Rizinus ${ }^{\circledR}$, erfolgen. Durch entsprechend ausgelöste Dehnungsreize soll so dem Darm die Chance gegeben werden, spontan eine möglicherweise anatomisch reversible Obstruktion zu überwinden. Zweitens sind bei distalen Motilitätsstörungen (Pseudoobstruktion des Kolons) Versuche lohnend, die Dickdarmmotilität mittels Einläufen (Hebe-SenkEinläufe) zu stimulieren [18, 21].

Ein anderer Ansatz zur Erhöhung der intestinalen Motilität besteht darin, den Abbau von Acetylcholin im synaptischen Spalt durch Hemmungen der Esterase zu reduzieren. Ein verfügbarer Acetylcholinesterasehemmer ist derzeit Neostigmin. Die intravenöse Applikation scheint dabei der intramuskulären überlegen zu sein. Allerdings ist Neostigmin bisher nicht zur Therapie von Motilitätsstörungen zugelassen (mit der Notwendigkeit eines «Off-Label Use»); zudem sind Kontraindikationen (chronisch obstruktive Lungenerkrankung) zu beachten. Bei intravenöser Applikation ist zusätzlich eine teilweise ausgeprägte Hypotonie- und Bradykardieneigung zu beachten, die gerade bei kritisch kranken Patienten unter Therapie ein intensives Monitoring erfordert [22].

Cholezystokinin kann ebenfalls zur medikamentösen Darmstimulation verwendet werden. Die Cholezystokininwirkung ist jedoch anatomisch unterschiedlich. Im oberen Gastrointestinaltrakt besteht eine Hemmung der Acetylcholinfreisetzung mit konsekutiver Verringerung der Motilität, während in weiter distalen Darmabschnitten die Acetylcholinfreisetzung und dadurch vor allem die Kolonmotilität relevant gesteigert werden können. Als Cholezystokinin-Agonist steht derzeit Sincalid zur Verfügung (mit der Notwendigkeit eines «Off-Label Use»). Bei der Anwendung ist ebenfalls eine teilweise ausgeprägte Bradykardie- und Hypotonieneigung zu berücksichtigen, die - ähnlich wie bei Neostigmin - unter i.v. Applikation eine engmaschige Überwachung des Patienten erfordert.

Bei der Pseudoobstruktion des Kolons handelt es sich um eine isolierte Überblähung des Kolons mit maximaler Distension der Zökalregion. Bei einer derartigen Kolonüberblähung von mehr als $10 \mathrm{~cm}$ Durchmesser ist zusätzlich zu den medikamentösen und mechanischen (Darmrohr) Maßnahmen eine endoskopische Therapie (Luftabsaugung) sowie in Extremfällen die Anlage einer Zökalfistel angezeigt [18].

\section{Disclosure Statement}

Die Autoren erklären hiermit, dass keine Interessenkonflikte bestehen. 
1 Hartl WH, Rittler P, Jauch KW: Metabolische und endokrine Besonderheiten beim operativen Intensivpatienten - Konsequenzen für die Therapie; in: Eckart J, Forst H, Buchardi H (Hrsg): Intensivmedizin. Landsberg/Lech, ecomed, 2003, XI-3, pp $1-28$.

2 Kreymann G, Adolph M, Druml W, Jauch KW; Working group for developing the guidelines for parenteral nutrition of The German Association for Nutritional Medicine: Intensive care medicine - guidelines on parenteral nutrition, Chapter 14 Ger Med Sci 2009;7:Doc14.

3 McClave SA, Martindale RG, Vanek VW, McCarthy M, Roberts P, Taylor B, Ochoa JB, Napolitano L, Cresci G; A.S.P.E.N. Board of Directors; American College of Critical Care Medicine; Society of Critical Care Medicine: Guidelines for the provision and assessment of nutrition support therapy in the adult critically ill patient: Society of Critical Care Medicine (SCCM) and American Society for Parenteral and Enteral Nutrition (A.S.P.E.N.). JPEN J Parenter Enteral Nutr 2009;33:277-316.

4 Singer P, Berger MM, Van den Berghe G, Biolo G Calder P, Forbes A, Griffiths R, Kreyman G, Leverve X, Pichard C; ESPEN: ESPEN Guidelines on Parenteral Nutrition: intensive care. Clin Nutr 2009;28:387-400.

5 Hartl WH, Muhl E: Stoffwechselveränderungen und Ernährungstherapie von Patienten nach großen viszeralchirurgischen Eingriffen und bei chirurgischen Intensivpatienten. Viszeralmedizin 2011; 27:28-40.

6 Hartl WH, Jauch KW, Parhofer K, Rittler P; Working group for developing the guidelines for parenteral nutrition of The German Association for Nutritional Medicine: Complications and monitoring - guidelines on parenteral nutrition, Chapter 11. Ger Med Sci 2009;7:Doc17.
7 Kreymann G, Adolph M, Mueller MJ; Working group for developing the guidelines for parenteral nutrition of The German Association for Nutritional Medicine: Energy expenditure and energy intake - guidelines on parenteral nutrition, Chapter 3. Ger Med Sci 2009;7:Doc25.

8 O'Keefe GE, Shelton M, Cuschieri J, Moore EE, Lowry SF, Harbrecht BG, Maier RV; Inflammation and the Host Response to Injury Collaborative Research Program: Inflammation and the host response to injury, a large-scale collaborative project: patient-oriented research core - standard operating procedures for clinical care VIII - nutritional support of the trauma patient. J Trauma 2008;65: 1520-1528.

9 Adolph M, Heller AR, Koch T, Koletzko B, Kreymann KG, Krohn K, Pscheidl E, Senkal M; Working group for developing the guidelines for parenteral nutrition of The German Association for Nutritional Medicine: Lipid emulsions - guidelines on parenteral nutrition, Chapter 6. Ger Med Sci 2009;7:Doc22.

10 Bolder U, Ebener C, Hauner H, Jauch KW, Kreymann G, Ockenga J, Traeger K; Working group for developing the guidelines for parenteral nutrition of The German Association for Nutritional Medicine: Carbohydrates - guidelines on parenteral nutrition, Chapter 5. Ger Med Sci 2009;7:Doc23.

11 Stein J, Boehles HJ, Blumenstein I, Goeters C, Schulz R; Working group for developing the guidelines for parenteral nutrition of The German Association for Nutritional Medicine: Amino acids guidelines on parenteral nutrition, Chapter 4. Ger Med Sci 2009;7:Doc24.

12 Weimann A, Ebener Ch, Holland-Cunz S, Jauch KW, Hausser L, Kemen M, Kraehenbuehl L, Kuse ER, Laengle F; Working group for developing the guidelines for parenteral nutrition of The German Association for Nutritional Medicine: Surgery and transplantation - guidelines on parenteral nutrition, Chapter 18. Ger Med Sci 2009;7:Doc10.
13 Mayer K, Weigand MA, Seeger W: Ernährung in der Intensivmedizin. Dtsch Med Wochenschr 2011; 136:1180-1183.

14 Weimann A, Andrä J, Sablotzki A: Ernährung bei Intensivpatienten. Dtsch Med Wochenschr 2011; 136:2251-2262.

15 Wernerman J: Combined enteral and parenteral nutrition. Curr Opin Clin Nutr Metab Care 2012; 15:161-165.

16 Weimann A, Jauch KW, Kemen M, et al.: Leitlinien enterale Ernährung - Chirurgie und Transplantation. Akt Ernährungsmed 2003;28(suppl 1): S51-S61.

17 Hartl WH, Kuppinger D: Monitoring der künstlichen Ernährung bei kritisch kranken Patienten. Intensivmedizin 2011;48:99-108.

18 Reintam Blaser A, Malbrain ML, Starkopf J, Fruhwald S, Jakob SM, De Waele J, Braun JP, Poeze M, Spies C: Gastrointestinal function in intensive care patients: terminology, definitions and management. Recommendations of the ESICM Working Group on Abdominal Problems. Intensive Care Med 2012;38:384-394.

19 Rittler P, Bolder U, Hartl WH, Jauch KW: Enterale Ernährung: Indikation und Zugangswege. Chirurg 2006;77:1063-1078.

20 Lübke HJ: Enterale Ernährung; in Eckart J, Forst $\mathrm{H}$, Buchardi $\mathrm{H}$ (Hrsg): Intensivmedizin. Landsberg/Lech, ecomed, 2003, XI-6, pp 1-22.

21 Bankhead R, Boullata J, Brantley S, Corkins M, Guenter P, Krenitsky J, Lyman B, Metheny NA, Mueller C, Robbins S, Wessel J; A.S.P.E.N. Board of Directors: Enteral nutrition practice recommendations. JPEN J Parenter Enteral Nutr 2009;33: 122-167.

22 Jauch KW, Hartl WH: Pharmatherapie der gastrointestinalen Insuffizienz; in Kuhlen R, Putensen C, Quintel M (Hrsg): Jahrbuch Intensivmedizin 2006. Berlin, Medizinisch Wissenschaftliche Verlagsgesellschaft, 2006, pp 237-256. 